\section{O Papel da Glicemia Capilar de Jejum no Diagnóstico Precoce do Diabetes Mellitus: Correlação com Fatores de Risco Cardiovascular}

\section{RESUMO}

O diabetes mellitus (DM) tem alta prevalência na população mundial. Para avaliar o papel da glicemia capilar de jejum (GCJ) no seu diagnóstico, 277 indivíduos (202F/75M) foram estudados. Os voluntários aparentavam ser saudáveis, tinham mais de 30 anos, e foram recrutados num posto de saúde quando estavam acompanhando pacientes ou se preparavam para fazer um exame de sangue de rotina. Gestação e conhecimento prévio de DM serviram de critérios de exclusão. Após avaliação antropométrica, a GCJ era medida. Os casos com GCJ >96mg/dl eram reavaliados com dosagens de glicemia plasmática em jejum e 2 horas após $75 \mathrm{~g}$ de dextrosol. Cento e oitenta e sete (67,5\%) tiveram GCJ s96mg/dl e 90 (32,5\%) GCJ >96mg/dl. Quando os parâmetros idade, peso, índice de massa corporal, cintura abdominal e quadril dos 2 grupos foram comparados entre si, os valores foram sempre estatisticamente maiores $(p<0,01)$ no grupo com $G C J>96 \mathrm{mg} / \mathrm{dll}$. A incidência de DM foi de $7,2 \%$ e de intolerância à glicose, 4,3\%. Em conclusão, a GCJ se mostrou um instrumento valioso no diagnóstico precoce do DM. (Arq Bras Endocrinol Metab 2002;46/3:255-259)

Descritores: Diabetes mellitus tipo 2; Glicemia capilar; Obesidade; Hiperglicemia

\begin{abstract}
The Role of Fasting Capillary Glycemia in the Diagnosis of Diabetes Mellitus: Correlation with Cardiovascular Risk Factors.

Diabetes mellitus (DM) is a very prevalent disease worldwide. To assess the role of fasting capillary glycemia (FCG) in the diagnosis of early DM, 277 subjects $(202 \mathrm{~F} / 75 \mathrm{M})$ were studied. Volunteers were apparently healthy, older than 30 years of age, and were recruited in a clinic where they came accompanying patients or to undergo a routine blood analysis. Pregnant women and patients with previous diagnosis of DM were excluded. After an anthropometric evaluation, a FCG was obtained. Cases with FCG $>96 \mathrm{mg} / \mathrm{dl}$ underwent fasting plasma glycemia repeated 2 hours after ingestion of $75 \mathrm{~g}$ dextrosol. FCG was $\leq 96 \mathrm{mg} / \mathrm{dl}$ in 187 cases $(67.5 \%)$, and $>96 \mathrm{mg} / \mathrm{dl}$ in 90 (32.5\%). When age, weight, body mass index, abdominal waist and hip/waist of the 2 groups were compared, values were all statistically higher $(p<0.01)$ in cases with FCG >96mg/dl. The incidence of DM was $7.2 \%$; glucose intolerance was present in $4.3 \%$. In conclusion, FCG was found to be a precious tool for the diagnosis of early DM. (Arq Bras Endocrinol Metab 2002;46/3:255-259)
\end{abstract}

Keywords: Type 2 diabetes mellitus; Capillary glycemia; Obesity; Hyperglycemia

\footnotetext{
A PREVALÊNCIA DO DIABETES MELLITUS (DM) na população adulta mundial é de cerca de $4 \%$ (1), podendo variar de acordo com a etnia e os hábitos de vida da população estudada, como alimentação, atividade física e prevalência de obesidade. Devido a diferenças no estilo e qualidade de vida das populações do mundo, existe, quando avaliamos isoladamente,
}

\section{artigo original}

\author{
Rubens A. Cruz Filbo \\ Livia Lugarinho Corrêa \\ Alessandra O. Ebrbardt \\ Gilberto Perez Cardoso \\ Gilberto Miranda Barbosa
}
Departamento de Medicina Clínica, Disciplina de Endocrinologia e Metabologia, Universidade Federal Fluminense, Niterói, RJ.


uma diferença marcante na prevalência do DM entre os países desenvolvidos (6\%) e os em desenvolvimento (3\%) (1). Em conseqüência destes fatos existiria uma forte tendência para o crescimento da prevalência do DM no mundo, sendo que de uma forma marcante nos países desenvolvidos, com uma previsão de prevalência para 2025 , de $7,6 \%(1)$.

Estima-se que cerca de $35 \%$ a $50 \%$ dos indivíduos com DM tipo 2 (DM2) não saibam ter a doença $(2,3)$, o que de forma significativa contribui, em muitos casos, para que já no diagnóstico clínico tenhamos a presença de alterações micro e macrovasculares. Se presumirmos que estas alterações vasculares geralmente aparecem, em média, 10 anos após o início da doença, fica bem claro a importância do seu diagnóstico precoce, além do que a vasculopatia é um importante fator causador de mortalidade e morbidade nesses indivíduos, podendo levar a insuficiência renal crônica, acidente vascular encefálico, doença coronariana, miocardiopatia, cegueira, lesão arterial dos membros inferiores e predisposição às infecções (3).

O DM2 é uma condição clínica geralmente insidiosa e que está associada com a obesidade na maioria dos casos (80-85\%) e é o tipo de DM mais freqüente em toda a população diabética, correspondendo a $90 \%$ das pessoas acometidas pela doença.

É de grande importância a realização deste trabalho em virtude da necessidade de se estabelecer o diagnóstico cada vez mais precoce do DM e de meios práticos de fazê-lo, para que se possa tentar, com o tratamento também iniciado precocemente, diminuir a morbi-mortalidade desta doença tão freqüente e crescente na população mundial (4).

\section{MATERIAL E MÉTODOS}

Foram selecionadas 277 pessoas de ambos os sexos (202F/75M) de forma aleatória em um posto de saúde do município de Niterói, trabalhando-se com um erro de amostragem de $3,2 \%$, que deveriam preencher os seguintes critérios: estarem em jejum, terem idade igual ou superior a 30 anos, não estarem grávidas e não possuírem história de DM. A proposta do estudo era apresentada às pessoas com possibilidade de participar e aqueles interessados eram submetidos à avaliação da glicemia capilar, avaliações antropométricas (peso, altura, cintura abdominal e quadril), mensuração da pressão arterial e respondiam às perguntas da ficha de avaliação: história familiar de DM e, se presente, o grau de parentesco, o tempo de diagnóstico e terapêutica utilizada, se era portador de alguma doença e sobre utilização de alguma medicação, além de dados pessoais, como data de nascimento, endereço, telefone e profissão.

A glicemia capilar de jejum (GCJ) era dosada através de um aparelho da marca Advantage ${ }^{\circledR}$ (Roche Diagnostics). Foi utilizada uma balança antropométrica mecânica da marca Filizola ${ }^{\circledR}$ para pesar e medir os participantes.

Não prosseguiam no estudo aqueles com resultados de GCJ $\leq 96 \mathrm{mg} / \mathrm{dl}$ e, conseqüentemente, prosseguiam aqueles com GCJ $>96 \mathrm{mg} / \mathrm{dl}$, sendo então encaminhados para a segunda fase do projeto.

Todos os participantes com GCJ $>96 \mathrm{mg} / \mathrm{dl}$ foram submetidos a exames laboratoriais que incluíam a avaliação da glicemia plasmática de jejum, glicemia 2 horas após $75 \mathrm{~g}$ de dextrosol, colesterol total, HDLcolesterol e triglicerídeos. Os critérios utilizados para o diagnóstico de DM e intolerância à glicose foram os estabelecidos em 1997 (5). Tanto os que tiveram o diagnóstico de DM confirmado (glicemia plasmática 2 horas após $75 \mathrm{~g}$ de dextrosol $\geq 200 \mathrm{mg} / \mathrm{dl}$ ), bem como os enquadrados no grupo de intolerantes à glicose (glicemia plasmática 2 horas após $75 \mathrm{~g}$ dextrosol $\geq 140$ e $<200 \mathrm{mg} / \mathrm{dl}$ ), estão sendo acompanhados pelo Serviço de Endocrinologia e Metabologia do Hospital Universitário Antônio Pedro em Niterói.

A análise estatística foi feita através da comparação das médias e desvios-padrão dos parâmetros avaliados (idade, peso, índice de massa corporal [IMC], circunferência abdominal e do quadril, GCJ, glicemia plasmática de jejum e 2 horas após $75 \mathrm{~g}$ de dextrosol) nos grupos com glicemia capilar $\leqslant 96 \mathrm{mg} / \mathrm{dl}$ e $>96 \mathrm{mg} / \mathrm{dl}$. Foi aplicado o teste $t$ de Student e adotado nível de significância de $5 \%$.

\section{RESULTADOS}

Do total de 277 pessoas avaliadas, $187(67,5 \%)$ tive$\mathrm{ram}$ GCJ $\leq 96 \mathrm{mg} / \mathrm{dl}$ e $90(32,5 \%) \mathrm{GCJ}>96 \mathrm{mg} / \mathrm{dl}$, sendo 58 mulheres e 32 homens. Dentre estes, 20 $(22,2 \%)$ tiveram o diagnóstico de DM confirmado, enquanto em $12(13,3 \%)$ os resultados foram compatíveis com intolerância à glicose e em $5(5,6 \%)$ o diagnóstico foi somente de intolerância de jejum (glicemia plasmática de jejum $\geq 110$ e $<126 \mathrm{mg} / \mathrm{dl}$ ). Dos 20 com o diagnóstico de DM, 11 (55\%) eram mulheres e $9(45 \%)$ homens; já no grupo de intolerantes à glicose, $10(83 \%)$ eram mulheres e $2(17 \%)$ homens e, finalmente, no grupo de indivíduos com somente intolerância de jejum, $3(60 \%)$ eram homens e $2(40 \%)$ mulheres. No total de 277 participantes, 20 $(7,2 \%)$ tiveram o diagnóstico de DM, $12(4,3 \%)$ into- 


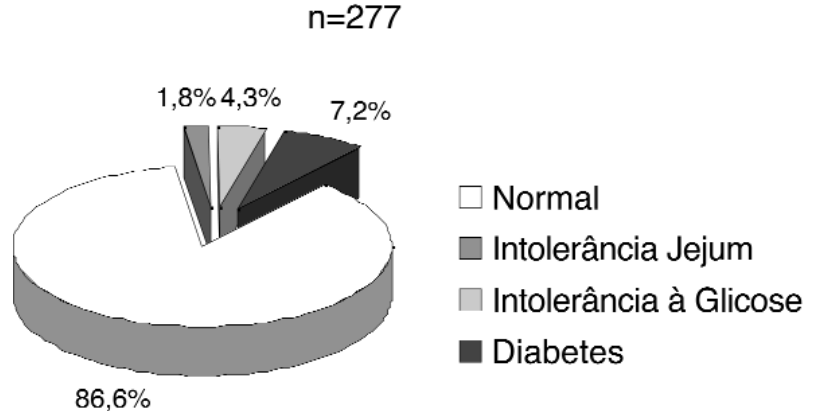

Figura 1. Proporção dos 277 indivíduos avaliados definidos em cada categoria diagnóstica.

lerância à glicose e $5(1,8 \%)$ intolerância de jejum (figura 1).

$\mathrm{Na}$ comparação das médias (e dp) entre os parâmetros avaliados em cada participante dos grupos (GCJ $>96$ e $\leq 96 \mathrm{mg} / \mathrm{dl}$ ), houve significância estatística $(\mathrm{p}<0,01)$ entre todos os parâmetros avaliados (tabela l). Ficou claramente demonstrado que os indivíduos do grupo com GCJ $>96 \mathrm{mg} / \mathrm{dl}$ eram mais velhos e tinham maiores IMC e cintura abdominal e do quadril (figura 2).

Já quando comparados os mesmos parâmetros, mas entre o grupo de indivíduos com diagnóstico de DM e o de intolerantes à glicose, não houve significância estatística (tabela 2), o que demonstrou uma semelhança no aspecto físico entre os indivíduos dos dois grupos, apenas diferindo no grau de tolerância à glicose.

Fazendo uma comparação entre o grupo de indivíduos com diagnóstico de DM e os com GCJ $<96 \mathrm{mg} / \mathrm{dl}$, verificamos nitidamente que os primeiros são mais velhos, pesados e com acúmulo predominantemente de gordura na região abdominal (tabela 3 ).

Tabela 1. Comparação dos parâmetros avaliados entre os grupos com GCJ $\leq 96 \mathrm{mg} / \mathrm{dl}$ e >96mg/dl.

\begin{tabular}{|c|c|c|c|}
\hline & $\begin{array}{c}\text { GCJ >96mg/dl } \\
\quad(n=90)\end{array}$ & $\begin{array}{c}\text { GCJ } \leq 96 \mathrm{mg} / \mathrm{dl} \\
(n=187)\end{array}$ & \multirow[t]{2}{*}{$p$} \\
\hline & Média \pm DP & Média \pm DP & \\
\hline Idade (anos) & $55 \pm 12$ & $47 \pm 13$ & $<0,01$ \\
\hline Peso (kg) & $75 \pm 15$ & $67 \pm 14$ & $<0,01$ \\
\hline $\mathrm{IMC}\left(\mathrm{kg} / \mathrm{m}^{2}\right)$ & $29 \pm 5$ & $26 \pm 5$ & $<0,01$ \\
\hline C. Abdominal $(\mathrm{cm})$ & $94 \pm 11$ & $87 \pm 11$ & $<0,01$ \\
\hline C. Quadril (cm) & $104 \pm 11$ & $99 \pm 11$ & $<0,01$ \\
\hline GCJ $(\mathrm{mg} / \mathrm{dl})$ & $129 \pm 60$ & $84 \pm 10$ & $<0,01$ \\
\hline Glicemia Jejum (mg/dl) & $115 \pm 50$ & & \\
\hline Glicemia $2 \mathrm{~h}(\mathrm{mg} / \mathrm{dl})$ & $142 \pm 77$ & & \\
\hline
\end{tabular}

GCJ: Glicemia capilar de jejum; IMC: Índice de massa corporal

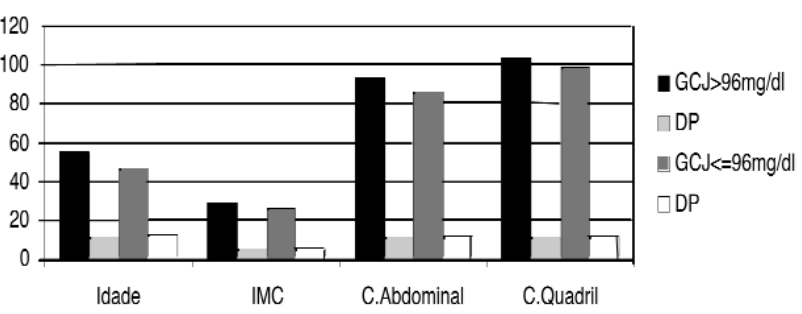

Figura 2. Parâmetros avaliados entre indivíduos com GCJ $>96 \mathrm{mg} / \mathrm{dl}$ e $\leq 96 \mathrm{mg} / \mathrm{dl}$.

Tabela 2 - Dados dos indivíduos com intolerância à glicose e com diagnóstico de diabetes.

\begin{tabular}{lccc}
\hline & $\begin{array}{c}\text { Intolerância } \\
\text { Glicose }(n=12)\end{array}$ & $\begin{array}{c}\text { Diabetes } \\
\text { Mellitus }(n=20)\end{array}$ & \\
\cline { 2 - 3 } & Média \pm DP & Média \pm DP & \\
\hline Idade (anos) & $58 \pm 12$ & $56 \pm 11$ & NS \\
IMC (kg/m²) & $29 \pm 4$ & $30 \pm 7$ & NS \\
C. Abdominal (cm) & $96 \pm 11$ & $96 \pm 13$ & NS \\
C. Quadril (cm) & $105 \pm 9$ & $105 \pm 15$ & NS \\
Peso (kg) & $72 \pm 14$ & $76 \pm 19$ & NS \\
\hline
\end{tabular}

IMC: Índice de Massa Corporal; NS: Não significativo

Tabela 3. Comparação dos parâmetros avaliados entre o grupo com diagnóstico de diabetes mellitus e com GCJ $\leq 96 \mathrm{mg} / \mathrm{dl}$.

\begin{tabular}{lccc}
\hline & $\begin{array}{c}\text { Diabetes } \\
\text { Mellitus }(\mathrm{n}=20)\end{array}$ & $\begin{array}{c}\text { GCJ } \leq 96 \mathrm{mg} / \mathrm{dl} \\
(\mathrm{n}=187)\end{array}$ & $\mathrm{P}$ \\
\cline { 2 - 3 } & Média $\pm \mathrm{DP}$ & Média $\pm \mathrm{DP}$ & \\
\hline Idade (anos) & $58 \pm 11$ & $47 \pm 13$ & $<0,01$ \\
Peso $(\mathrm{kg})$ & $72 \pm 19$ & $67 \pm 14$ & $<0,01$ \\
IMC $\left(\mathrm{kg} / \mathrm{m}^{2}\right)$ & $30 \pm 7$ & $26 \pm 5$ & $<0,05$ \\
C. Abdominal (cm) & $96 \pm 13$ & $87 \pm 11$ & $<0,01$ \\
C. Quadril (cm) & $105 \pm 15$ & $99 \pm 11$ & $\mathrm{NS}$ \\
\hline
\end{tabular}

GCJ: Glicemia capilar jejum; IMC: Índice de massa corporal; NS: Não significativo

Quando avaliamos o perfil lipídico dos indivíduos com diagnóstico de DM e aqueles com intolerância à glicose, verificamos que nos primeiros os triglicerídeos estavam significativamente mais elevados $(204 \mathrm{mg} / \mathrm{dl} \pm 216)$, talvez já evidenciando uma alteração na lipase lipoprotéica, enzima dependente da presença de insulina. Por outro lado, no grupo dos intolerantes foram encontrados níveis mais elevados de colesterol total e LDL-colesterol $(\mathrm{p}<0,01)$. O HDLcolesterol, no entanto, era idêntico nos dois grupos (tabela 4). 
Tabela 4. Perfil lipídico nos grupos com diabetes e intolerantes à glicose.

\begin{tabular}{lccc}
\hline & $\begin{array}{c}\text { Diabetes } \\
\text { Mellitus }(n=20)\end{array}$ & $\begin{array}{c}\text { Intolerância } \\
\text { Glicose }(n=12)\end{array}$ & $p$ \\
\cline { 2 - 3 } & Média \pm DP & Média \pm DP & \\
\hline Colesterol Total (mg/dl) & $217 \pm 34$ & $225 \pm 61$ & $<0,01$ \\
HDL-Colesterol (mg/dl) & $44 \pm 11$ & $43 \pm 10$ & NS \\
LDL-Colesterol (mg/dl) & $140 \pm 29$ & $149 \pm 57$ & $<0,01$ \\
Triglicerídeos (mg/dl) & $204 \pm 216$ & $163 \pm 77$ & $<0,01$ \\
\hline
\end{tabular}

NS: Não significativo

\section{DISCUSSÃO}

O DM2 é uma doença com prevalência muito elevada em todo o mundo e com projeções epidêmicas para os próximos anos $(1,3)$. As complicações em decorrência da evolução desta doença, especificamente naqueles que perduram durante anos com alterações na tolerância à glicose, mas sem diagnóstico e sem o devido tratamento, tornam clara a importância do diagnóstico precoce e a necessidade da implementação do tratamento adequado, com mudanças nos hábitos alimentares e no estilo de vida o mais precocemente possível. Sem falarmos na possibilidade cada vez mais clara de se tentar evitar a evolução do intolerante à glicose para o DM propriamente dito $(6,7)$.

A utilização da GCJ como método de screening, ou mesmo de diagnóstico, como feito neste estudo, demonstrou agilidade, praticidade, rapidez e segurança, como deve ser um método de screening ideal (8), na detecção dos indivíduos que deveriam ser investigados. Os resultados (figura 1) com uma taxa de prevalência no grupo de indivíduos estudados de 7,2\% para DM, 4,3\% intolerância à glicose e $1,8 \%$ intolerância de jejum, por si só demonstram a importância do método de seleção empregado. Nós utilizamos como ponto de corte o valor da GCJ $>96 \mathrm{mg} / \mathrm{dl}$, diferentemente do estudo canadense (9), em que foi utilizado um valor para glicemia capilar casual $>99 \mathrm{mg} / \mathrm{dl}$, para que aí sim os indivíduos fossem melhor avaliados. Talvez por isso a prevalência de novos casos de DM tenha sido baixa $(2,2 \%)$ nesse estudo, quando comparada com o nosso $(7,2 \%)$. Não podemos deixar de levar em consideração que o estudo canadense foi de âmbito nacional com 9.042 indivíduos avaliados. Entretanto, o fato de termos definido $96 \mathrm{mg} / \mathrm{dl}$ como o valor de ponto de corte para a GCJ, além de termos estudado os indivíduos em jejum, conferiu maior rigidez ao nosso estudo, talvez por isso permitindo que tivéssemos uma maior prevalência tanto para DM quanto para intolerância à glicose.
Se levarmos em consideração que: (1) grande parte dos indivíduos com diagnóstico de DM e também aqueles com intolerância à glicose, ou mesmo intolerância de jejum, estavam assintomáticos, ou com sinais e sintomas ainda não perceptíveis, pelo menos para o paciente, e (2) a maioria tinha glicemia plasmática de jejum normal, nós poderíamos classificá-los como indivíduos que tinham alteração na tolerância à glicose $\mathrm{e}$ não sabiam e conseqüentemente não tratavam. Como sabemos dos riscos da hiperglicemia crônica para o aparecimento de lesões micro e macrovasculares a longo prazo, fica clara a importância da detecção e tratamento precoces das alterações da tolerância à glicose, como também fica claro o porquê de muitos indivíduos ao diagnóstico de DM2 já apresentarem complicações micro e macrovasculares (10-12).

Os indivíduos com GCJ >96mg/dl e, entre eles, aqueles com diagnóstico de DM, tinham maior peso e IMC, além de maior circunferência abdominal, quando comparados com indivíduos com GCJ $\leqslant 96 \mathrm{mg} / \mathrm{dl}$ (tabela 1 e 3). Este fato associa diretamente a adiposidade com alterações na tolerância à glicose, como também abre a possibilidade para fortes especulações e mesmo evidências da participação do tecido adiposo como órgão endócrino secretor de polipeptídios, no caso específico da resistina, leptina, fator de necrose tumoral alfa (TNF-a), adipsina e Acrp30/adipoQ, além de ácidos graxos livres, todos com provável papel fundamental no desenvolvimento da resistência insulínica e conseqüentemente no aparecimento do DM2 (13). Entretanto, a evolução fisiopatológica do DM2 não se caracteriza somente pela presença da resistência insulínica, mas também por uma disfunção na secreção da mesma (14), o que não invalida, mas até acrescenta, o fato do DM2 tanto em humanos quanto em modelos de roedores estar fortemente associado com resistência insulínica $(15,16)$. Contudo, ainda não está claro, apesar das fortes evidências, a correlação da obesidade com a resistência insulínica (13).

A importância do método de screening e do conseqüente achado de alteração na tolerância à glicose em quase $14 \%$ dos indivíduos avaliados demonstra que este tipo de procedimento de detecção pode e deve ser empregado com maior intensidade dentro de nossa sociedade. Somente o fato de 7,2\% dos indivíduos terem DM2 e não saberem já justifica qualquer argumento. O achado de 4,3\% dos indivíduos com intolerância à glicose também é uma forte justificativa, além do que se adicionarmos o fato desses indivíduos intolerantes terem maiores valores de LDL-colesterol e de colesterol total, a justificativa fica ainda mais nítida. A que grau de agressão do sistema vascular esses indi- 
víduos não estavam sendo submetidos? Também não podemos deixar de lembrar da possibilidade de prevenção da evolução de um quadro de intolerância para um de DM. Alguns estudos têm demonstrado a possibilidade de se interromper esta evolução com mudanças nos hábitos e estilo de vida (alimentação, atividade física, emagrecimento) (6,7).

A utilização da GCJ em indivíduos com mais de 30 anos e utilizando-se o valor de GCJ $>96 \mathrm{mg} / \mathrm{dl}$ como ponto de corte para maior investigação se mostrou um bom método para detecção de indivíduos com alteração na tolerância à glicose.

\section{AGRADECIMENTOS}

Gostaríamos de agradecer ao CNPq/PIBIC/UFF pelo apoio financeiro a este projeto; ao professor Pedro Carvalho Rodrigues pela ajuda com a orientação estatística; à Produtos Roche Químicos e Farmacêuti$\cos$ S. A. pela doação do aparelho Advantage e das tiras utilizadas para obtenção da glicemia capilar e ao professor Jocemir Ronaldo Lugon pela ajuda e orientação crítica.

\section{REFERÊNCIAS}

1. King H, Aubert RE, Herman WH. Global burden of diabetes, 1995-2025: prevalence, numerical estimates, and projections. Diabetes Care 1998:21:1414-31.

2. Harris Ml, Eastman RC. Early detection of undiagnosed diabetes mellitus: a US perspective. Diabetes Metab Res Rer 2000; 16:230-6.

3. Engelgau MM, Narayan KMV, Herman WH. Screening for type 2 diabetes. Diabetes Care 2000;23:1563-80.

4. Roman SH, Harris Ml. Management of diabetes mellitus from a public health perspective. Endocrinol Metab Clin N Am 1997;26:443-74.

5. Expert committee on the diagnosis and classification of diabetes mellitus: Report of the expert committee on diagnosis and classification of diabetes mellitus. Diabetes Care 1997;20:1183-97.
6. Toumilehto J, Lindström J, Eriksson JG, Valle $\Pi$, Hämäläinen $\mathrm{H}$, llanne-Parikka $\mathrm{P}$, et al. Prevention of type 2 diabetes mellitus by changes in lifestyle among subjects with impaired glucose tolerance. N Engl J Med 2001;344:1343-50.

7. Swinburn BA, Metcalf PA, Ley SJ. Long-term (5-year) effects of a reduced-fat diet intervention in individuals with glucose intolerance. Diabetes Care 2001;24:619-24.

8. Engelgau MM, Aubert RE, Thompson TJ, Herman WH. Screening for NIDDM in nonpregnant adults: a review of principles, screening tests, and recommendations. Diabetes Care 1995;18:1606-18.

9. Leiter LA, Barr A, Bélanger A, Lubin S, Ross SA, Tildesley $\mathrm{HD}$ et al. Diabetes screening in Canada (DIASCAN) study. Diabetes Care 2001;24:1038-43.

10. Rajala U, Laakso M, Qiao Q, Keinanen-Kiukaanniemi S. Prevalence of retinopathy in people with diabetes, impaired glucose tolerance, and normal glucose tolerance. Diabetes Care 1998;21:1664-9.

11. Kohner EM, Aldington SJ, Stratton IM, Manley SE, Holman RR, Matthews DR, et al. United Kingdom prospective diabetes study: diabetic retinopathy at diagnosis of non-insulin-dependent diabetes mellitus and associated risk factors. Arch Ophthalmol 1998; 1 16:297-303.

12. Harris Ml, Klein R, Welborn TA, Knuiman MW. Onset of NIDDM occurs at least 4-7 years before clinical diagnosis. Diabetes Care 1992; 15:815-9.

13. Steppan CM, Bailey ST, Bhat S, Brown EJ, Banerjee RR, Wright CM, et al. The hormone resistin links obesity to diabetes. Nature 2001;409:307-12.

14. Taylor Sl. Deconstructing type 2 diabetes. Cell 1999; 97:9-12.

15. Kopelman PG. Obesity as a medical problem. Nature 2000;404:635-43.

16. Matthael S, Stumvoli M, Kellerer M, Häring H. Pathophysiology and pharmacological treatment of insulin resistance. Endocrine Rev 2000;21:585-618.

\section{Endereço para correspondência:}

Rubens Antunes da Cruz Filho

Rua Visconde de Moraes, 126/802

24210-145 Niterói, RJ

FAX: (021) 2704-5300

e.mail: radcf@urbi.com.br 